\title{
As empresas da Região Metropolitana do Recife e a exploração de SI/TI
}

\author{
Ana Paula Cabral Seixas Costa \\ José Gilson de Almeida Teixeira Filho \\ Maisa Mendonça Silva \\ UFPE
}

\begin{abstract}
Resumo
Este artigo apresenta os primeiros resultados obtidos com uma investigação sobre a exploração de Sistemas de Informação (SI) e Tecnologia da Informação (TI) em 37 (trinta e sete) empresas da Região Metropolitana do Recife (RMR), sendo estas em sua maioria empresas privadas, que atuam no ramo de serviço, com faturamento anual médio abaixo de $\mathrm{R} \$ \mathbf{5 0 0} .000,00$. Este estudo exploratório tem por objetivo familiarizar os pesquisadores com a realidade do uso de SI/TI nesta região e permitir a construção de hipóteses sobre o tema.
\end{abstract}

\section{Palavras-chave}

Sistemas de Informação, Tecnologia da Informação, Investimento em Tecnologia da Informação.

\section{The companies of metropolitan area of Recife and the exploration of SI/TI}

\begin{abstract}
This article presents the first results obtained with an investigation about the exploration of Information Systems (IS) and Information Technology (IT) in 37 (thirty seven) companies of the Metropolitan Area of Recife (RMR), being these in your majority deprived companies, that they act in the service branch, with medium annual billing below $R \$ 500.000,00$. This exploratory study has for objective to familiarize the researchers with the reality of the use of IS/IT in this area and to allow the construction of hypotheses about theme.
\end{abstract}

Key words

Information Systems, Information Technology, Information Technology Investment. 


\section{INTRODUĈ̣̃O}

O presente artigo descreve a pesquisa desenvolvida junto ao setor produtivo da Região Metropolitana do Recife (RMR) sobre como e em que nível as empresas dessa região exploram e dependem de Sistemas de Informação e Tecnologia da Informação. Desenhando um cenário sobre qual é o estágio de desenvolvimento tecnológico em sistemas de informação dessas empresas e construindo algumas hipóteses a partir do estudo deste ambiente.

Como mencionado, o campo de investigação é a Região Metropolitana do Recife (RMR), onde foram pesquisadas empresas de indústria, comércio e serviços.

O trabalho busca responder se as empresas da RMR percebem o potencial benefício que os SI/TI podem trazer para o negócio e exploram esse potencial de forma a obter vantagens competitivas, além de identificar se essas empressas carecem de metodologias para planejar e decidir sobre os investimentos em SI/TI de forma a melhor explorá-los. inclusive a necessidade de definição de novos modelos de referência.

Neste trabalho desenvolveu-se uma pesquisa na Região Metropolitana do Recife (RMR), que é formada por algumas das cidades mais populosas do Estado de Pernambuco como: Recife (1.421.993), Jaboatão dos Guararapes (580.795), Olinda (368.666), Paulista (262.072).

Pernambuco tem hoje um PIB de R\$ 24,7 bilhões, correspondendo este valor a 2,71\% do PIB brasileiro e a $17,1 \%$ do PIB do Nordeste. Este PIB apresenta-se dividido da seguinte forma: Serviços, 68\%; Indústria, 24,7\%; Agropecuária, 6,4\%.

Os principais ramos industriais são: produtos alimentares, química, material elétrico e de comunicação, bebidas, metalúrgica, minerais não-metálicos e agroindústrias.

O Estado dispõe de onze Distritos Industriais, sendo oito na Região Metropolitana do Recife (RMR) e três no interior do Estado. Na Região Metropolitana, os Distritos estão localizados nos municípios de Paulista, Abreu e Lima, Centro Urbano do Curado (Jaboatão), Itapissuma, São Lourenço da Mata, Cabo e o Multifabril de Jaboatão, além do Parqtel - Distrito Industrial destinado a empresas de Base Tecnológica.

Os principais ramos de serviços são: saúde, informática, bancos e distribuição. Entre os setores de ponta encontra-se o pólo de infor-

Com o crescente papel dos Sistemas de Informação (SI) e da Tecnologia da Informação (TI) no desempenho e na estratégia das organizações, pesquisas de campo foram realizadas em vários países do mundo, buscando diagnosticar como as organizações amadureceram com relação à exploração de $\mathrm{SI} / \mathrm{TI}$ nas diversas perspectivas que envolvem este tema. Estas pesquisas resultaram em modelos que são hoje citados como referência na literatura, como os modelos de Nolan e Gibson (1974), Sullivan (1985), além de conclusões sobre aspectos que influenciam o sucesso da exploração de SI/TI nas organizações tais como: perfil de profissionais gestores da empresa e gestores de SI/TI; critérios para avaliação de projetos e decisão de investimento em SI/TI; a importância do Planejamento de SI/TI para o correto investimento em SI/TI; os fatores críticos de sucesso para este Planejamento, entre outras nuanças.

Entretanto são poucos os registros no Brasil de pesquisas que retratem o perfil das nossas empresas com relação à exploração de SI/TI, não sendo possível verificar se os vários modelos e conclusões encontradas na literatura se aplicam às realidades brasileiras (considerando que existem diferenças significativas entre as várias regiões do Brasil). Uma pesquisa de campo nesta área pode mostrar cenários completamente distintos daqueles encontrados nos países da Europa e nos Estados Unidos, implicando mática conhecido como Porto Digital.

A indústria de software em Recife é responsável por $1 \%$ do PIB do Estado de Pernambuco e gera algo em torno de 1000 empregos, com 58 empresas de alta tecnologia.

O pólo recifense da indústria de software do país, também chamado de Porto Digital, é hoje um dos principais pólos no Brasil, dessa indústria que apresenta um crescimento de $11 \%$ ao ano, desenvolvendo soluções em Tecnologia da Informação (SCHELP, 2003).

A implantação do Porto Digital consolidou Pernambuco como Pólo Tecnológico e influenciou ações de várias empresas internacionais tais como: a instalação do escritório de vendas para o Nordeste dos produtos da Internet e a implantação da WeDo Soft da holding portuguesa Sonae no Recife.

\section{ESTUDOS EXPLORATÓRIOS SOBRE SISTEMAS DE INFORMAC̄ÃO E TECNOLOGIA DA INFORMAC̣̃̃O}

Uma pesquisa publicada no Information \& Management (CLAVER et al., 2000) mostra a relevância de pesquisas na área de Sistemas de Informação, analisando os tópicos mais freqüentemente abordados nos periódicos Information \& Management e MIS Quartely, no período de 1981 
a 1997. Entre as várias publicações encontradas, Claver et al. (2000) relata que parte delas dizem respeito a pesquisas de campo que buscam diagnosticar cenários com relação aos vários aspectos que envolvem o uso dos Sistemas de Informação. Em suas conclusões o autor comenta que tem aumentado o número de artigos empíricos sobre os teóricos. Entre os mais freqüentes estudos empíricos está a pesquisa de campo, seguida do estudo de caso. É possível encontrar na literatura várias pesquisas de campo que buscam melhor entender como têm sido percebidos e utilizados os Sistemas de Informações nas organizações.

Entre estes estudos empíricos, encontram-se pesquisas sobre: variáveis e critérios que afetam a decisão de usar ou não SI; como é feito o planejamento e seleção de SI; o impacto do ambiente e dos gestores no planejamento e uso de SI; a relação entre vantagem competitiva e TI, entre outros aspectos. Algumas dessas pesquisas relatadas na literatura são sintetizadas a seguir.

\section{As decisões de investimento em SI/TI}

Thong (1999) apresenta em seu artigo uma pesquisa com 166 pequenas empresas e discute como características dos executivos (CEO - Chief Executive Officer) e da própria organização podem afetar a decisão de uma empresa de investir ou não em Sistemas de Informação. Nas conclusões do autor, empresas com CEOs que apresentam características inovadoras e algum conhecimento sobre Sistemas de Informação apresentam maior probabilidade de realizarem o investimento. Da mesma forma, características da organização como o tamanho do negócio, corpo de empregados com conhecimento em Sistemas de Informação e a intensidade do fluxo de informação também contribuem para a realização do investimento.

Proudlock et al. (1998) realizaram uma pesquisa exploratória tomada em empresas na Inglaterra, focando como as empresas decidem sobre adotar Sistemas de Informação e como realizam a seleção dos produtos de software, investigando a influência dos recursos internos das organizações, do time de gerentes, considerado escasso em pequenas empresas, tamanho da organização e ambiente.

Perez (1998) investigou os critérios utilizados pelas empresas para tomar a decisão de investimentos, concluindo que estes são bastante diversos. Segundo Perez (1998), $42,1 \%$ das empresas espanholas utilizam como critério a necessidade de SI que respondam aos SI dos competidores, $92,1 \%$ das empresas consideram como critério mais importante para decidir pelo investimento a capacidade de apoiar a tomada de decisão gerencial. Esses são critérios relacionados com a gestão, mas critérios relacionados a requerimentos técnicos também são utilizados. Perez (1998) mostra que, em média, 39,2\% das organizações utilizam requerimentos técnicos para avaliar os projetos. Outro dado interessante é que, nos últimos anos, o aprendizado de novas tecnologias não aparecia como um fator relevante a ser considerado no investimento. Atualmente, esse mesmo critério é aplicado por $57,9 \%$ das empresas. Também são aplicados os critérios financeiros, uma vez que $86,84 \%$ das organizações utilizam pelo menos um critério financeiro na avaliação de seus projetos de SI (PEREZ, 1998).

Um estudo realizado por Li e Ye (1999) discute e empiricamente testa os efeitos do ambiente dinâmico, estratégia da firma e organização de $\mathrm{CEO} / \mathrm{CIO}$ (Chief Information Officer) sobre o impacto do investimento em TI, e este último sobre o desempenho de uma organização. Como resultado, o investimento em TI aparece tendo um forte e positivo impacto sobre o desempenho financeiro de organizações que estão inseridas em ambientes de muitas mudanças, com estratégias mais proativas, e nas quais existe um link estreito entre CEOs e CIOs. Eles concluem também que as organizações que consideram o investimento em TI devem conhecer o contexto do ambiente em que estão inseridas, suas direções estratégicas, e a alta gerência deve permitir ao CIOs desempenhar um papel mais estratégico.

Burn e Szeto (2000) em sua pesquisa assumem que o efetivo alinhamento de TI e a estratégia do negócio podem ser atingidos por meio do planejamento de SI. Esses autores realizaram um estudo usando o modelo de Henderson e Vankatraman e pesquisaram em várias indústrias as perspectivas das duas visões: organização e área de TI. Na maioria delas, essas perspectivas são equivalentes, mas algumas diferenças foram encontradas. A conclusão é que a visão do alinhamento estratégico varia primeiramente com a natureza da organização e depois com a dependência quanto a TI.

A pesquisa entrevistou gestores organizacionais (CEOs - Chief Enterprise Officers) e gestores de SI/TI (CIOs - Chief Information Officers) de empresas de exportação e importação, atacadistas, seguros, bancos, transportes, comunicação e outras. Quatro perspectivas de alinhamento estratégico foram consideradas: Execução da Estratégia, Transformação da Tecnologia, Potencial Competitivo e Nível de Serviço. Os resultados mostraram que as percepções dos CIOs e CEOs são similares com relação à direção, às necessidades e aos princípios do alinhamento entre as estratégias do negócio e a TI.

As divergências são encontradas na percepção dos problemas na implementação desse alinhamento. Ambos os grupos indicam que a estratégia do negócio deve conduzir a estratégia de alinhamento, que o papel da alta gerência é priorizar e encontrar a melhor forma de alocar os recursos dentro das organizações, e que o papel dos gerentes de SI deve ser de liderança executiva, guiando a função de TI de modo a alcançar os objetivos estratégicos.

Sobre a importância e a dificuldade de realizar planejamento de SI, vários resultados de pesquisa podem ser 
encontrados. Entre eles, em seu trabalho, Teo e King (1997) concluem que a integração entre o plano de negócio e o plano de SI pode ser definida em termos do movimento por meio de quatro tipos de integração: administrativa, seqüencial, recíproca e completa. Nesse trabalho foi percebido que apenas poucas organizações indicaram ter usado a integração completa.

- Integração Administrativa - nesse tipo de integração existe uma fraca relação entre plano de negócio e plano de SI. O que há são planos separados com integração administrativa. Geralmente existe um pequeno, embora significativo, esforço no uso de TI para suportar o plano de negócio.

- Integração Seqüencial - nesse tipo de integração, o plano de negócio provê direções para o plano de SI. O foco do plano de SI é dar suporte ao plano de negócio. O alinhamento entre os planos acontece em apenas um sentido.

- Integração Recíproca - nesse tipo de integração existe uma recíproca e interdependente relação entre o plano de negócio e o plano de SI. O plano de SI desempenha ambos os papéis, de suportar e influenciar o plano de negócio. O alinhamento entre os planos acontece nos dois sentidos.

- Integração Completa - nesse tipo de plano, existe uma pequena diferença entre o processo de planejamento do negócio e o processo de planejamento de SI. Contudo, estratégias de negócio e de SI são desenvolvidas no mesmo processo integrado de planejamento.

Em sua pesquisa, Teo e King (1997) concluíram que, sob uma perspectiva evolucionária, as empresas apresentam diferentes níveis de integração e passam de um nível para outro, e que apenas poucas empresas conseguem a integração completa.

Uma grande preocupação dos envolvidos com o planejamento de SI é entender os fatores que são críticos para o seu sucesso: Teo e Ang (1999) realizaram uma pesquisa em 169 empresas, dentre elas, bancos, empresas de seguros, químicas, telecomunicações, informática, engenharia, arquitetura, hotéis, agências de viagens, indústrias, atacadistas. Encontraram como principais fatores críticos de sucesso para o planejamento:

- Alta gerência comprometida com o uso estratégico de TI;

- Gestores de SI conhecedores do negócio;

- Gestores de Negócio e SI trabalharem juntos em parceria na priorização das aplicações;

- Objetivo de o negócio ser conhecido pelos gestores de SI;

- A área de SI ser sensível às necessidades dos usuários.

Numa investigação contínua, Teo e Ang (2001), em pesquisa realizada, dentre os principais problemas para o insucesso do planejamento de SI nas organizações, apresentam:
- Ausência de ativa participação e envolvimento da alta gerência - essa participação garante o conhecimento dos objetivos estratégicos da organização e o alinhamento do plano de SI com esses objetivos, tornando esse plano realmente eficaz.

- Falhas na transferência dos objetivos e estratégias em planos de ação.

- Negligência no ajuste do plano de SI como reflexo de mudanças no ambiente.

Pode-se encontrar também pesquisas sobre como, face a importância estratégica dos SI para as organizações, estas devem decidir em quais projetos de SI/TI fazer seu investimento. Segundo Jiang e Klein (1999), a seleção de SI/TI envolve vários passos, incluindo seleção e ponderação das alternativas. Escolha e ponderação de critérios cruciais na seleção dos projetos, e não simplesmente a aplicação de uma técnica financeira tradicional.

Para alocar recursos escassos em diversos projetos de SI, profissionais de SI freqüentemente necessitam conduzir um processo de avaliação do potencial desses projetos. O critério de avaliação inclui rotineiramente aspectos tecnológicos, econômicos e comportamentais.

$\mathrm{Na}$ literatura, são descritos vários métodos para a seleção de projetos, em geral divididos em quatro subcategorias (JIANG; KLEIN, 1999): Abordagem comparativa; Modelo de scoring; Modelo Econômico; Métodos de Otimização.

Como já é consenso na literatura que os investimentos feitos em SI afetam o desempenho de uma organização, as discussões sobre esse tema continuam cada vez mais freqüentes (DHILLON; HACKNEY, 2003; CROWSTON; MYERS, 2004; TIERNAN; PEPPARD, 2004; HU; QUAN, 2005). Irani (2002), citando o trabalho de outros autores, sugere que o desempenho financeiro de uma organização está mais relacionado com os recursos de SI que são gerenciados do que com os investimentos feitos pela organização em novas tecnologias. Não é surpresa que surjam questões de como os investimentos no portfólio de SI são avaliados, gerenciados e controlados, e ainda a complexidade de gerir os benefícios intangíveis e custos indiretos. Esses problemas e questões têm sido largamente reportados na literatura. Como resultado, temas comuns que têm implicações na avaliação de SI têm sido discutidos, tais como (IRANI, 2002):

- Entender o mecanismo humano e o organizacional e o processo de tomada de decisão de investimento dentro das organizações;

- Disponibilizar a melhor tecnologia e integração de sistemas de negócio;

- Entender o conceito de valor e suas multidimensionais facetas;

- Descobrir as políticas associadas a orçamento e tomada de decisão; 
- Navegar através da taxonomia de benefício do investimento;

- Conhecer a natureza dos benefícios de SI/TI (intangíveis, tangíveis, financeiros e não-financeiros);

- Identificar, gerir e controlar o custo do investimento (direto e indireto);

- Avaliar o portfólio de investimentos por meio de técnicas adequadas;

- Conhecer os riscos associados às diferentes estratégias de investimento;

- Entender o escopo e o impacto do desenvolvimento de uma infra-estrutura de TI;

- Entender a complexidade da avaliação incremental do desenvolvimento, integração e melhoria de sistemas;

- Definição de Stakeholders, análise e envolvimento (inclusive cultura);

- Prover adequada tecnologia para a gestão dos recursos. as relações entre os SI/TI e o negócio e o papel dos CIO e CEO no ambito dessas relações (MUATA et al., 2003; RANGANATHAN \& KANNABIRAN, 2004; RONDEAU et al., 2005; COUGLHAN et al., 2005; CHEN et al., 2005).

\section{O planejamento de $\mathrm{SI} / \mathrm{TI}$}

Percebe-se então que, desde o início dos anos 90, melhorar o processo de planejamento de SI tem sido uma das principais preocupações dos gestores de SI, tendo como principal objetivo da melhoria desse processo garantir o alinhamento entre os planos de negócio e SI, conscientes de que, para o sucesso desse planejamento, esse alinhamento é fundamental.

Uma abordagem diferente na busca desse alinhamento foi apresentada por Croteau e Bergeron (2001). Eles estudaram empiricamente como um perfil de desenvolvimento tecnológico específico para um tipo de estratégia de negócio pode dar melhor suporte ao desempenho organizacional.

Irani (2002) argumenta que os altos índices de falhas nos investimentos em SI são particularmente atribuídos à ausência de sólidas ferramentas de uso gerencial para avaliar, priorizar, monitorar e controlar os investimentos de SI. Um

\section{- ste trabalho busca familiarizar o pesquisador — com a realidade do uso de SI/TI na RMR através de uma pesquisa exploratória.} outro argumento para justificar as

falhas é que em muitas organizações são usadas apenas justificativas econômicas como foco para a decisão de investimento.

Irani (2002) explica que, quando o propósito do investimento em SI é melhorar a eficiência operacional, muitas técnicas tradicionais de avaliação podem ser consideradas apropriadas. Contudo, muitos gerentes buscam no investimento o suporte para as estratégias do negócio, então surge o problema: como calcular, quantificar e acomodar as implicações de um investimento em SI dentro dos métodos de avaliação tradicionais? Por fim, Irani (2002) conclui que a tomada de decisão de investimento é um complexo processo gerencial, especialmente devido ao escopo e magnitude da interação sociotécnica das variáveis, que nem sempre podem ser quantificadas em termos financeiros. Ainda como resultado do seu trabalho de pesquisa, o autor afirma que:

Custos indiretos dos projetos de SI são freqüentemente mais significativos que seus custos diretos e podem ser classificados como tendo as dimensões humana e organizacional.

Os benefícios podem ser classificados como estratégico, tático e operacional, com natureza financeira, não-financeira e intangível.

Existe uma grande variedade de interação de fatores sociais e técnicos que complicam o processo de avaliação. Neste contexto podem ser encontradas pesquisas que investigam
Definem em seu trabalho a Estratégia de Negócio como sendo o resultado da decisão tomada para guiar uma organização com respeito ao ambiente, estrutura e processos que influenciam seu desempenho organizacional.

O Desenvolvimento Tecnológico corresponde à maneira como as empresas planejam e gerenciam a TI, para conseguirem benefícios potenciais e efetividade.

Segundo Croteau e Bergeron (2001), medir o desempenho organizacional pode ser um problema, visto que não existe universalmente nenhuma medida que descreva esse conceito. Ainda segundo os autores, o desempenho organizacional pode ser medido usando-se dados objetivos e subjetivos. A abordagem objetiva refere-se aos dados financeiros da organização. A abordagem subjetiva depende da percepção de quem está fazendo o julgamento.

No trabalho são definidos quatro tipos de estratégia empresarial: prospecção, análise, defesa e reação.

As firmas escolhem um tipo ou outro de acordo com a percepção que têm do ambiente onde estão inseridas. Espera-se que os primeiros três tipos aumentem o desempenho organizacional e compartilhem o mesmo contínuo no qual a estratégia de prospecção está num extremo, a defesa no outro e a estratégia de análise está no centro. Na opinião desses autores, espera-se que a estratégia de reação impeça o desempenho organizacional, sendo ela excluída do contínuo no qual estão as outras. 
Organizações apoiadas por estratégias de prospecção almejam acesso a uma grande fatia de mercado. Elas são caracterizadas por seus repetidos esforços para inovar e trazer mudanças para sua produção. No outro extremo, organizações favorecidas por uma estratégia de defesa têm um mercado restrito, produção eficiente, enfatizam a excelência dos seus produtos, a qualidade dos seus serviços e seus baixos preços.

As organizações que implementam estratégias de análise compartilham as características das organizações que optaram pelas duas estratégias anteriores, mas moderadamente. Finalmente, as organizações apoiadas por estratégias de reação ignoram suas oportunidades e não podem manter mercados prontos para aquisição ou correr riscos verdadeiros.

Os resultados do estudo de Croteau e Bergeron (2001) mostram, como já era esperado, que existe um link positivo entre as estratégias de defesa, análise, prospecção e desempenho organizacional, e um link negativo entre esse e as estratégias de reação. Os autores concluem que diferentes perfis de desenvolvimento tecnológico podem contribuir para as estratégias de prospecção, análise e reação. No entanto, um perfil específico de desenvolvimento tecnológico contribui indiretamente para o desempenho organizacional de empresas que adotaram as estratégias de prospecção, enquanto outro perfil de desenvolvimento tecnológico contribui diretamente para o desempenho organizacional de empresas que optaram pelas estratégicas de análise. $\mathrm{Na}$ opinião dos autores, esses resultados indicam que as organizações podem aumentar seu desempenho com apoio de estratégias de prospecção e análise e desenvolvimento de TI.

Em outra pesquisa realizada sobre o tema, desta vez focando fatores que impactam no planejamento de SI, Basu et al. (2002) pesquisaram em 105 corporações quanto ao impacto do comprometimento organizacional, envolvimento da alta gerência e envolvimento do time de planejamento no planejamento de Sistemas de Informação estratégicos.

Segundo Basu et al. (2002), o planejamento de Sistemas de Informações estratégicos é uma importante função gerencial, que pode ajudar uma organização a usar a TI mais competitivamente, identificar novas aplicações de TI e melhor prever as necessidades de recursos de TI. Por outro lado, as falhas no planejamento podem causar perdas de oportunidades e duplicação de esforços. Podem resultar, também, em incompatibilidade de sistemas e perdas de recursos. Hoje, os ambientes altamente competitivos e as mudanças rápidas na TI agravam os perigos da inefetividade do planejamento muito mais que antes.

Em seu trabalho, Basu et al. (2002) avaliam o impacto positivo e negativo sobre o planejamento de SI em relação às seguintes variáveis:

- Comprometimento organizacional - suporte da companhia para o planejamento;
- Envolvimento da alta gerência;

- Envolvimento do time de planejamento - o time de planejamento inclui ambos os gerentes usuários e os profissionais de Sistemas de Informação.

Basu et al. (2002) ainda afirmam que o processo de planejamento de SI numa organização tem como função determinar um portfólio de aplicações baseadas em computador, as quais ajudem a organização a atingir seus objetivos de negócios.

Segundo esses autores, o sucesso do planejamento de SI pode ser medido pela extensão do alcance dos objetivos a que esse planejamento se propõe e que podem ser muitos:

- Ajudar a organização a ganhar vantagem competitiva;

- Ajudar a definir novas estratégias de negócio;

- Facilitar a gestão e o controle dos recursos de TI;

- Identificar a arquitetura e a política de tecnologia;

- Aumentar a visibilidade de TI na organização.

Nesse estudo, eles percebem que o insuficiente envolvimento da alta gerência pode comprometer o sucesso do planejamento, e também confirmaram que o excessivo comprometimento organizacional pode igualmente comprometer o sucesso do planejamento, porque muitos recursos disponíveis para ser gerenciados podem criar atrasos burocráticos. Como conclusão, argumenta-se que nem sempre muito planejamento significa melhor planejamento.

Byrd e Turner (2001) apresentam os resultados de uma investigação sobre o relacionamento entre infra-estrutura de Tecnologia da Informação e vantagem competitiva e argumentam que nas últimas duas décadas Tecnologia da Informação tem sido apresentada como um dos recursos que as organizações podem usar para obter vantagem competitiva, e que ao mesmo tempo o valor competitivo da TI vem dos Sistemas de Informação Estratégicos para as organizações, estes mudam os objetivos, as operações, os produtos ou o relacionamento das organizações com o ambiente para ajudá-las a obter vantagens sobre os competidores. Em seu trabalho, Byrd e Turner (2001) obtiveram a opinião dos gerentes de TI de 1.000 empresas, buscando confirmar se existe uma relação entre fatores que caracterizam flexibilidade da infra-estrutura de TI e medidas associadas com vantagens competitivas.

Andersen (2001), baseado em questionário enviado a 180 empresas nos EUA, investiga como o uso de Sistemas de Informação em rede está associado com o desempenho das organizações e, em contrapartida, como um ambiente de tomada de decisão descentralizado pode favorecer a associação acima e as diferenças dessas hipóteses entre empresas mais e menos dinâmicas e complexas.

Como mencionado, a despeito destes estudos encontrados na literatura, são poucos os registros no Brasil de pesquisas 
que retratem o perfil das nossas empresas com relação à exploração de SI/TI. Neste contexto, este trabalho busca familiarizar o pesquisador com a realidade do uso de SI/TI na RMR através de uma pesquisa exploratória.

\section{METODOLOGIA DA PESQUISA}

Para iniciar a pesquisa procurou-se primeiramente entender qual tipo de metodologia seria necessário para construir hipóteses sobre o ambiente que se deseja explorar. Percebeu-se que seria interessante, de acordo com o tema, focar o trabalho em uma entrevista, porém a entrevista implicaria em tempos e custos elevados. Assim, em vez da entrevista, optou-se por realizar uma observação direta extensiva, que nada mais é que realizar por meio de questionário, de formulário, de medidas de opinião e atitudes ou de técnicas mercadológicas uma observação de determinado aspecto (MARCONI; LAKATOS, 2002). Nesta pesquisa o instrumento utilizado foi o questionário.

Marconi e Lakatos (2002) definem o questionário como um instrumento de coleta de dados constituído por uma série de perguntas, que devem ser respondidas por escrito e sem a presença do entrevistador. Em geral, o pesquisador envia o questionário ao informante, pelo correio ou por portador; depois de preenchido, o pesquisado devolve-o do mesmo modo.

Ainda segundo Marconi e Lakatos (2002), o pesquisador deve enviar uma nota ou carta explicando a natureza da pesquisa, sua importância e a necessidade de obter respostas, tentando despertar o interesse do recebedor para que ele preencha e devolva o questionário dentro de um prazo razoável. As vantagens de se utilizar esse tipo de instrumento no auxílio à coleta de dados para a pesquisa são: abrange uma área geográfica mais ampla; obtém respostas mais rápidas e mais precisas; há menos risco de distorção, pela não influência do pesquisador; há mais tempo para responder e em hora mais favorável. Por outro lado, tem-se como desvantagens: percentagem pequena dos questionários que voltam (em média 25\%); grande número de perguntas sem resposta; a dificuldade de compreensão, por parte dos informantes, leva a uma uniformidade aparente; a devolução tardia prejudica o calendário ou sua utilização.

O questionário deve conter de 20 a 30 perguntas e demorar no máximo cerca de 30 minutos para ser respondido. Em relação às questões, estas devem ser codificadas, a fim de facilitar, mais tarde, a tabulação (MARCONI; LAKATOS, 2002). Nesta pesquisa, através do questionário destinado a gestores de empresas da RMR, foram inseridas 17 (dezessete) perguntas com o objetivo de tornar mínimo o tempo de preenchimento, visto que as pessoas em geral não desejam ocupar-se por muito tempo respondendo a muitas perguntas. Como o foco desta pesquisa eram os gestores de empresas, e para estes em especial o tempo despendido para responder à pesquisa é considerado fator crítico, reduziu-se o número de perguntas e estimou-se gastar em torno de 6 (seis) a 10 (dez) minutos para o preenchimento do questionário, e todas as questões foram codificadas para facilitar a análise e tabulação dos dados.

Após a elaboração do questionário, foi realizado o Teste de Instrumentos e Procedimentos de Pesquisa, o Pré-Teste, em que o questionário é testado antes de sua utilização definitiva, aplicando-se alguns exemplares em uma pequena população escolhida, que neste caso foi a turma de alunos do IV curso de especialização em gestão da informação da UFPE - Universidade Federal de Pernambuco. Após a análise dos dados do pré-teste, é possível verificar falhas existentes no instrumento de pesquisa, tais como: inconsistência ou complexidade das questões; ambigüidade ou linguagem inacessível; perguntas supérfluas ou que causem embaraço ao informante.

\section{ma pesquisa de campo na área de TI pode daqueles encontrados em outros países.}

O que foi percebido rapidamente na primeira aplicação do questionário é que faltavam questões mais específicas com perguntas mais técnicas e outras mais dirigidas aos CEOs (Chief Executive Officer) das organizações.

O questionário apresentado no Quadro 1 foi denominado: Pesquisa sobre o perfil das empresas em relação ao uso de Sistemas de Informação (SI) e Tecnologia da Informação (TI). O foco do questionário de empresas foi direcionado a obter informações de faturamento, percentual de investimentos em TI, características do gestor, percepção da função TI na empresa, exploração da TI. A área da pesquisa também foi citada para facilitar a compreensão e a percepção do leitor de imediato e levá-lo a entender a abrangência da pesquisa assim como se segue: Área de aplicação: Região Metropolitana do Recife - Período: 2을 Semestre de 2004 e 1을 Semestre de 2005. Segundo Marconi e Lakatos (2002) as perguntas, em geral, utilizadas no questionário são classificadas em fechadas (limitadas ou de alternativas fixas, são aquelas em que o informante escolhe sua resposta entre duas opções: sim e não) e de múltipla escolha (fechadas, mas que apresentam uma série de possíveis respostas, abrangendo várias facetas do mesmo assunto).

Com o objetivo de obter um maior número de respondentes e facilitar o preenchimento do mesmo, o questionário foi 
implementado também em meio digital, sendo construído um site na Internet. Assim, tornou-se menos onerosa a pesquisa, pois os respondentes ficaram com mais uma opção à disposição para responder a pesquisa, além de todos os dados já serem automaticamente armazenados em um banco de dados.

Para análise dos dados obtidos foi utilizado o software STATISTICA StatSoft (2001). Foram realizados os seguintes procedimentos de análise: estatística descritiva, análise de regressão e teste de hipótese. Neste artigo é apresentado apenas parte dos primeiros resulatdos obtidos com o estudo explorário.

\section{ANÁLISE E INTERPRETAC̣ÃO DOS DADOS}

Como resultado da pesquisa constata-se que em um universo total de 37 (trinta e sete) empresas pesquisadas, 31 (trinta e uma empresas) são privadas e 6 (seis) são públicas, como apresentado na Tabela 4.1 a seguir. Percebe-se também que em relação ao ramo de atividade as empresas pesquisadas são: 30 (trinta) a maioria, prestadoras de serviços, 5 (cinco) indústrias e 2 (duas) empresas de comércio.

A Tabela 4.1 mostra que a grande maioria das empresas respondentes são empresas privadas que atuam no ramo de serviços.

Tabela 4.1: Ramo de atividade e tipo de empresa.

\begin{tabular}{|l|c|c|c|}
\hline \multirow{2}{*}{$\begin{array}{c}\text { RAMO DE } \\
\text { ATIVIDADE }\end{array}$} & \multicolumn{2}{|c|}{ TIPO DE EMPRESA } & \multirow{2}{*}{ TOTAL } \\
\cline { 2 - 3 } & PRIVADA & PÚBLICA & \\
\hline Serviços & 24 & 6 & 30 \\
\hline Indústria & 5 & 0 & 5 \\
\hline Comércio & 2 & 0 & 2 \\
\hline Total & 31 & 6 & 37 \\
\hline
\end{tabular}

Fonte: os autores
A Tabela 4.2 mostra a faixa de faturamento anual das empresas pesquisadas por ramo de atividade. Pode-se observar que das 37 (trinta e sete) empresas pesquisadas, apenas 30 (trinta) responderam a esse item, concentrando-se as respostas obtidas em empresas com faturamento anual abaixo de $\mathrm{R} \$ 500.000,00$ e atuando no ramo de serviços.

$\mathrm{Na}$ pesquisa foram incluídas questões para avaliar como a TI é utilizada nas organizações. Estas questões descrevem de forma adaptada para os dias atuais os seis estágios de crescimento com relação a exploração de SI/TI propostos no modelo de Nolan e Gibson (1979). A Tabela 4.3 mostra os resultados encontrados na pesquisa. As organizações passam por fases ou estágios de maturação em relação ao uso da TI - Tecnologia da Informação. O primeiro estágio representa uma falta excessiva de utilização da tecnologia para auxiliar a tomada de decisões e o controle de hardware e software da organização. Conforme a organização avança em relação à maturação no uso da tecnologia começa a haver uma clara integração entre os sistemas existentes, gerenciamento dos recursos de informação, planejamento bem estabelecido e alinhamento dos SI - Sistemas de Informação com a organização.

Abaixo é apresentado o modelo proposto por Nolan e Gibson (1979), que sugere seis estágios de crescimento com relação à exploração de SI/TI. Estes estágios, como já foi mencionado, são descritos de forma adaptada para os dias atuais:

I - Iniciação - Controle descentralizado e mínimo planejamento. Uso de computadores por pequeno número de usuários para atender necessidades básicas da organização.

II - Contágio - Disseminação das aplicações. Utilização de computadores por um grande número de usuários e crescimento ao acaso sem nenhum controle central.

III - Controle - Centralização. Controle de custos.

IV - Integração - Previsão de benefícios potenciais, integrando os sistemas existentes, compartilhando dados, aplicações. Redes ou outras ferramentas apropriadas são necessárias para realizar a proposta. Os gastos crescem novamente.

Tabela 4.2. Faixa de faturamento por ramo de atividade.

\begin{tabular}{|c|c|c|c|c|}
\hline \multirow{2}{*}{$\begin{array}{c}\text { FAIXA DE FATURAMENTO BRUTO } \\
\text { ANUAL DA EMPRESA }\end{array}$} & \multicolumn{3}{|c|}{ RAMO DE ATIVIDADE } & \multirow[b]{2}{*}{ TOTAL } \\
\hline & SERVIÇO & INDÚSTRIA & COMÉRCIO & \\
\hline Abaixo de $\mathrm{R} \$ 500.000,00$ & 11 & 2 & 1 & 14 \\
\hline De $R \$ 500.000,01$ a $R \$ 1.000 .000,00$ & 1 & 1 & 0 & 2 \\
\hline De $R \$ 1.000 .000,01$ a $R \$ 5.000 .000,00$ & 5 & 1 & 0 & 6 \\
\hline De $\mathrm{R} \$ 10.000 .000,01$ a $\mathrm{R} \$ 50.000 .000,00$ & 6 & 1 & 0 & 7 \\
\hline Acima de $\mathrm{R} \$ 50.000 .000,00$ & 1 & 0 & 0 & 1 \\
\hline Total & 24 & 5 & 1 & 30 \\
\hline
\end{tabular}

Fonte: os autores 
V - Administração de Dados - Estabelecimento de controles de gerenciamento sobre os recursos de informação. Isto difere do estágio de controle porque os controles prévios são aplicados apenas aos produtos (hardware e software) próprios.

VI - Maturidade. Integração das aplicações. Controles ajustados. Planejamento bem estabelecido. Alinhamento dos sistemas de informação com a organização.

Dessa maneira pode-se dizer que a empresa que se encontra no estágio seis explora em todas as sua potencialidades SI e TI, podendo usufruir e obter mais vantagens competitivas em relação a outras organizações que ainda não alcançaram esta maturidade.
Pelos resultados encontrados na Tabela 4.3 não é possível afirmar que as empresas estão agrupadas em determinado estágio.

A Tabela 4.4 mostra que a maioria das empresas de serviços e privadas investem em TI anualmente menos de R $\$$ $50.000,00$, e uma análise de regressão linear simples para empresas privadas pesquisadas, relacionando o faturamento anual médio das empresas e o investimento anual médio em TI, apresentada na Tabela 4.5, mostra que o investimento em TI explica em torno de $40 \%$ do faturamento destas empresas.

Entretanto, como algumas das empresas pesquisadas são do setor de informática, estas poderiam estar enviesando o resultado da análise, portanto, para confirmar os resultados

Tabela 4.3. Estágios de utilização de TI.

\begin{tabular}{|l|c|c|c|c|}
\hline \multirow{2}{*}{$\begin{array}{c}\text { ESTÁGIOS DE } \\
\text { NOLAN E GIBSON }\end{array}$} & \multicolumn{3}{c|}{ RAMO DE ATIVIDADE } & TOTAL \\
\cline { 2 - 5 } & SERVIçO & INDÚSTRIA & COMÉRCIO & 7 \\
\hline Estágio I & 3 & 2 & 2 & 5 \\
\hline Estágio II & 4 & 1 & 0 & 8 \\
\hline Estágio III & 8 & 0 & 0 & 6 \\
\hline Estágio IV & 5 & 1 & 0 & 2 \\
\hline Estágio V & 2 & 0 & 0 & 9 \\
\hline Estágio VI & 8 & 1 & 2 & 37 \\
\hline Total & 30 & 5 & & \\
\hline
\end{tabular}

Fonte: os autores

Tabela 4.4: Investimento anual em SI/TI por ramo de atividade e tipo de empresa.

\begin{tabular}{|c|c|c|c|c|c|}
\hline \multirow{2}{*}{$\begin{array}{c}\text { QUANTO A EMPRESA INVESTE } \\
\text { ANUALMENTE EM SI/TI }\end{array}$} & \multirow{2}{*}{$\begin{array}{c}\text { TIPO DE } \\
\text { EMPRESA }\end{array}$} & \multicolumn{3}{|c|}{ RAMO DE ATIVIDADE } & \multirow{2}{*}{ TOTAL } \\
\hline & & SERVIÇO & INDÚSTRIA & COMÉRCIO & \\
\hline \multirow{3}{*}{ Abaixo de $\mathrm{R} \$ 50.000,00$} & Privada & 14 & 3 & 2 & 19 \\
\hline & Pública & 2 & 0 & 0 & 2 \\
\hline & Total & 16 & 3 & 2 & 21 \\
\hline \multirow{3}{*}{ De $\mathrm{R} \$ 50.000,01$ a $\mathrm{R} \$ 100.000,00$} & Privada & 4 & 1 & 0 & 5 \\
\hline & Pública & 1 & 0 & 0 & 1 \\
\hline & Total & 5 & 1 & 0 & 6 \\
\hline \multirow{3}{*}{ De R\$ 100.000,01 a R\$ 500.000,00 } & Privada & 3 & 1 & 0 & 4 \\
\hline & Pública & 0 & 0 & 0 & 0 \\
\hline & Total & 3 & 1 & 0 & 4 \\
\hline \multirow{3}{*}{ De $R \$ 500.000,01$ a $R \$ 1.000 .000,00$} & Privada & 1 & 0 & 0 & 1 \\
\hline & Pública & 3 & 0 & 0 & 3 \\
\hline & Total & 4 & 0 & 0 & 4 \\
\hline \multirow{3}{*}{ Acima de $\mathrm{R} \$ 5.000 .000,00$} & Privada & 2 & 0 & 0 & 2 \\
\hline & Pública & 0 & 0 & 0 & 0 \\
\hline & Total & 2 & 0 & 0 & 2 \\
\hline
\end{tabular}

Fonte: os autores 
da Tabela 4.5 acima, realizou-se uma outra regressão retirando agora da análise as empresas de informática. $\mathrm{O}$ resultado é apresentado na Tabela 4.6 a seguir.

Observa-se que os resultados sofreram pouca alteração, não invalidando as conclusões anteriores.
Na Tabela 4.7 pode-se observar que a maioria das empresas apresenta um nível de automação (grau em que os processos desenvolvidos na empresa são automatizados por TI) médio e não utiliza metodologia para fazer planejamento de sistemas de informação.

Tabela 4.5: Regressão linear simples.

\begin{tabular}{|c|}
\hline SUMÁRIO DE REGRESSÃo PARA A VARIÁVEL DEPENDENTE: FATURAMENTO ANUAL \\
\hline Variável independente: Investimento anual médio em TI \\
\hline $\mathrm{R}=, 65480454 \quad \mathrm{R}^{2}=, 42876899 \quad$ Adjusted $\mathrm{R}^{2}=, 40280395$ \\
\hline $\mathrm{F}(1,22)=16,513 \mathrm{p}<, 00052$ Std. Error of estimate: $4670 \mathrm{3}$ \\
\hline
\end{tabular}

Fonte: os autores

Tabela 4.6: Análise de regressão com empresas privadas que não são do setor de informática.

\begin{tabular}{|c|}
\hline SUMÁRIO DE REGRESSÃO PARA A VARIÁVEL DEPENDENTE: FATURAMENTO ANUAL \\
\hline Variável independente: Investimento anual médio em TI \\
\hline $\mathrm{R}=, 65088279 \quad \mathrm{R}^{2}=, 42364841 \quad$ Adjusted $\mathrm{R}^{2}=, 38762643$ \\
\hline $\mathrm{F}(1,22)=11,76083 \mathrm{p}<, 003440$ \\
\hline
\end{tabular}

Fonte: os autores

Tabela 4.7: Metodologia de planejamento e nível de automação.

\begin{tabular}{|c|c|c|c|c|c|c|}
\hline \multirow{2}{*}{$\begin{array}{l}\text { RAMO DE } \\
\text { ATIVIDADE }\end{array}$} & \multirow{2}{*}{$\begin{array}{l}\text { TIPO DE } \\
\text { EMPRESA }\end{array}$} & \multirow{2}{*}{$\begin{array}{l}\text { A EMPRESA UTILIZA } \\
\text { ALGUMA METODOLOGIA DE } \\
\text { PLANEJAMENTO DE SI }\end{array}$} & \multicolumn{3}{|c|}{$\begin{array}{l}\text { QUAL O NÍVEL DE AUTOMAÇÃO EM } \\
\text { QUE A EMPRESA SE ENCONTRA }\end{array}$} & \multirow{2}{*}{ TOTAL } \\
\hline & & & MÉDIO & BAIXO & ALTO & \\
\hline \multirow{6}{*}{ Serviço } & \multirow{2}{*}{ Privada } & Não & 11 & 0 & 4 & 15 \\
\hline & & Sim & 1 & 3 & 5 & 9 \\
\hline & Total & & 12 & 3 & 9 & 24 \\
\hline & \multirow{2}{*}{ Pública } & Não & 3 & 0 & 1 & 4 \\
\hline & & Sim & 1 & 0 & 1 & 2 \\
\hline & Total & & 4 & 0 & 2 & 6 \\
\hline \multirow{6}{*}{ Indústria } & \multirow{2}{*}{ Privada } & Não & 1 & 2 & 0 & 3 \\
\hline & & Sim & 1 & 0 & 1 & 2 \\
\hline & Total & & 2 & 2 & 1 & 5 \\
\hline & \multirow{2}{*}{ Pública } & Não & 0 & 0 & 0 & 0 \\
\hline & & Sim & 0 & 0 & 0 & 0 \\
\hline & Total & & 0 & 0 & 0 & 0 \\
\hline \multirow{6}{*}{ Comércio } & \multirow{2}{*}{ Privada } & Não & 0 & 1 & 0 & 1 \\
\hline & & Sim & 1 & 0 & 0 & 1 \\
\hline & Total & & 1 & 1 & 0 & 2 \\
\hline & \multirow{2}{*}{ Pública } & Não & 0 & 0 & 0 & 0 \\
\hline & & Sim & 0 & 0 & 0 & 0 \\
\hline & Total & & 0 & 0 & 0 & 0 \\
\hline
\end{tabular}

Fonte: os autores 
Realizou-se uma avaliação dos dados para verificar o grau de difusão das empresas com relação ao uso de TI segundo o modelo de Sullivan (1985). Como métrica utilizou-se a relação entre o número de computadores e número de funcionários fornecido pelas empresas. Aquelas empresas que apresentaram esta relação com valor superior a 0,5 , o que significa superior a dois funcionários por computador, foram consideradas de alta de difusão, as demais de baixa difusão. O resultado desta análise é apresentado na tabela 4.8. A maioria da empresas pesquisadas, privadas ou públicas, apresentam alta difusão.

Com relação ao grau de infusão, ainda segundo o modelo de Sullivan (1985), utilizaram-se como métrica as respostas às questões: Como a empresa se encontra com relação a cultura de sistemas de informação; Qual o nível de dependência da empresa em relação ao uso de sistemas de informação e a disponibilidade das informações para auxiliar uma tomada de decisão de diretoria. Os seguintes critérios de avaliação do grau de infusão, apresentado na Tabela 4.9, foram adotados.

Os resultados desta análise são apresentados na Tabela 4.10.

A maioria das empresas pesquisadas apresenta baixa infusão. As duas últimas análises indicam que, embora façam uso de computadores em toda a organização, a TI não penetrou nas empresas em termos de impacto e importância.

Uma análise das características das empresas que apresentam alta difusão permite concluir que nestas empresas os gestores percebem a TI como diferencial competitivo (em alguns casos são identificadas além desta, outras percepções.) e são utilizados ao menos critérios técnicos para decidir sobre o investimento em TI.

Prosseguindo com a exploração dos dados realizou-se um teste de hipótese e verificou-se uma correlação estatisticamente significativa entre a variável percepção que o gestor da empresa tem de TI e o critério utilizado pela empresa para decidir sobre o investimento em TI. Dos respondentes, $37,84 \%$ percebem a TI como uma ferramenta para melhoria dos processos internos, como um diferencial competitivo e como um facilitador da comunicação - destes, 50\% utilizam critérios técnicos, critérios financeiros, tendências de mercado para decidir sobre o investimento em TI; $29,73 \%$ percebem a TI apenas como uma ferramenta para melhoria dos processos internos - destes, $54,55 \%$ utilizam critérios técnicos para decidir sobre o investimento em TI; $16,22 \%$ dos respondentes percebem a TI como diferencial competitivo - destes, $66,67 \%$ usam critérios técnicos para decidir

Tabela 4.8: Nivel de Difusão.

\begin{tabular}{|l|c|c|l|c|}
\hline \multicolumn{1}{|c|}{ TIPO EMPRESA } & \multicolumn{2}{c|}{ PUBLIGA } & \multicolumn{2}{c|}{ PRIVADA } \\
\hline RAMO ATIVIDADE & ALTA DIFUSÃOO & BAIXA DIFUSÃO & ALTA DIFUSÃOO & BAIXA DIFUSÃO \\
\hline Serviço & 4 & 2 & 16 & 7 \\
\hline Industria & 0 & 0 & 3 & 2 \\
\hline Comércio & 1 & 0 & 0 & 1 \\
\hline
\end{tabular}

Fonte: os autores

Tabela 4.9: Nivel de Infusão.

\begin{tabular}{|l|l|l|}
\hline & \multicolumn{1}{|c|}{ ALTA INFUSÃO } & \multicolumn{1}{c|}{ BAIXA INFUSÃ̃ } \\
\hline Cultura de sistema de informação & Difundida entre todos os setores & Nenhuma ou em fase inicial \\
\hline Nível de dependência & Alto & Baixo ou médio \\
\hline Disponibilidade de informação & Fácil acesso & Difícil ou médio acesso \\
\hline
\end{tabular}

Fonte: os autores

Tabela 4.10: Resultados nível difusão/infusão.

\begin{tabular}{|l|c|c|c|c|}
\hline \multicolumn{1}{|c|}{ TIPO EMPRESA } & \multicolumn{2}{c|}{ PUBLICA } & \multicolumn{2}{c|}{ PRIVADA } \\
\hline \multicolumn{1}{|c|}{ RAMO ATIVIDADE } & ALTA INFUSÃOO & BAIXA INFUSÃO & ALTA INFUSÃOO & BAIXA INFUSÃO \\
\hline ServiçO & 0 & 5 & 7 & 18 \\
\hline Industria & 0 & 0 & 2 & 3 \\
\hline Comércio & 0 & 0 & 0 & 2 \\
\hline
\end{tabular}


sobre o investimento em TI; $8,11 \%$ percebem a TI apenas como facilitador da comunicação - destes, $66,67 \%$ utilizam critérios financeiros para decidir sobre investimento.

Conclui-se que as empresas cujos gestores percebem a TI como uma possibilidade para melhoria interna dos processos e diferencial competitivo utilizam para tomar a decisão sobre investimento em TI critérios técnicos, critérios financeiros e tendências de mercado. As empresas cujos gestores percebem a TI apenas como facilitador da comunicação utilizam critérios financeiros para decidir sobre o invesimento em TI ou outros critérios não especificados na resposta à pesquisa.

Quando perguntadas sobre a utilização de ferramentas de TI de apoio a gestão e tomada de decisão tais como ERP, CRM, Data mining e Data warehouse, $44,44 \%$ das empresas responderam não utilizar nenhuma dessas ferramentas e 17\% responderam utilizar ERP e CRM. O sistema operacional de rede mais utilizado entre as empresas pesquisadas é Microsoft Windows (62\%), o Banco de Dados é o Oracle (30\%) e as ferramentas de desenvolvimento mais utilizadas são Delphi, Java e ASP. Com respeito às tecnologias de Internet a maioria das empresas pesquisadas tem site institucional e ou uma intranet.

\section{CONCLUSÃO}

Este artigo apresenta os primeiros resultados obtidos com uma investigação sobre a a exploração de SI/TI em 37 (trinta e sete) empresas da Região Metropolitana do Recife, sendo estas em sua maioria empresas privadas, que atuam no ramo de serviços, com faturamento anual médio abaixo de R $\$ 500.000,00$. Este estudo exploratório tem por objetivo familiarizar os pesquisadores com a realidade do uso de TI nesta região e permitir a construção de algumas hipóteses. Como o objetivo é construir hipóteses e não testá-las, o tamanho da amostra pesquisada não é crítico. Com a análise dos primeiros resultados observa-se que estas empresas investem anualmente em TI menos que $\mathrm{R} \$ 50.000,00$ (o correspondente a aproximadamente $1 \%$ do seu faturamento anual) e que, no entanto, em especial para as empresas privadas, o investimento em TI explica algo em torno de $40 \%$ do faturamento destas empresas. Em geral as empresas apresentam um nível médio de automação e não utilizam metodologias para realizar planejamento de TI. Outro resultado obtido é que a percepção que o gestor da empresa tem de TI reflete-se nos critérios e na forma utilizada para decidir sobre o investimento em TI. Todos estes resultados corroboram o encontrado na literatura em outros estudos exploratórios (THONG, 1999; PEREZ, 1998; LI; YE, 1999; IRANI, 2002).

Como conclusão geral a pesquisa indica que estas empresas ainda não têm consciência do benefício que a exploração do potencial de TI pode trazer para o negócio e carecem de metodologias para planejar e decidir sobre a exploração deste potencial, além de ferramentas para apoiar a gestão. Como prosseguimento desta pesquisa serão realizados novos estudos exploratórios, com um número maior de empresas, objetivando testar as hipóteses construídas com esta primeira investigação.

\section{Artigo recebido em 15/06/2005 Aprovado para publicação em 23/05/2006}

\section{- Referências Bibliográficas}

ANDERSEN, T. J. Information technology, strategic decision making approaches and organizational performance in different industrial settings. Journal of Strategic Information Systems, n. 10, p. 101-110, 2001.

BASU, V. et al. The impact of organizational commitment, senior management involvement, and team involvement on strategic information systems planning. Information \& Management, n. 39, p. 513524, 2002.

BURN, J. M.; SZETO, C. A. Comparison of the views of business and IT management on success factor for strategic alignment. Information \& Management, n. 37, p. 197216, 2000.
BYRD, T. A.; TURNER, D. E. An exploratory examination of the relationship between flexible IT infrastructure and competitive advantage. Information \& Management, $\mathrm{n}$. 39, p. 41-52, 2001.

CHEN, H. H. G.; MILLER, R.; JIANG, J. J.; KLEIN, G. Communication skills importance and pro.ciency: perceptiondifferences between IS staff and IS users. International Journal of Information Management. n. 25, p. 215-227, 2005.

CLAVER, E.; GONZÁLES, R.; LLOPIS, J. An analysis of research in information systems. Information \& Management, $\mathrm{n}$. 37 , p. $181-195,2000$
COUGHLAN, J.; LYCETT, M.; MACREDIE, R.D. Understanding the business-IT relationship. International Journal of Information Management, n. 25, p. 303319, 2005.

CROTEAU ANNE-MARIE; BERGERON F. An information technology trilogy: business strategy, technological deployment and organization performance. Journal of Strategic Information Systems, n. 10, p. 77-99, 2001.

CROWSTON, K.; MAYERS, M. D. Information technology and the transformation of industries: three research perspectives. Journal of Strategic Information Systems, n. 13 , p. $5-28,2004$
DHILLON, G.; HACKNEY, R. Positioning IS/IT in networked firms. International Journal of Information Management, n. 23 , p. 163-169, 2003.

HU, Q.; QUAN, J. J. Evaluating the impact of IT investments on productivity:a causal analysis at industry level. International Journal of Information Management, n. 25 p. 39-53, 2005.

IRANI ZAHIR. Information systems evaluation: navigating through the problem domain. Information \& Management, n. 40, p. 11-24, 2002 
- Referências Bibliográficas

LI, M.; YE, L. R. Information technology and firm performance: Linking with environmental, strategic and managerial contexts. Information \& Management, $\mathrm{n}$. 35, p. 43-51, 1999.

MARCONI, M. A.; LAKATOS, E. M. Técnicas de Pesquisa: planejamento e execução de pesquisas, amostragens e técnicas de pesquisas, elaboração, análise e interpretação de dados. 5. ed. São Paulo: Atlas, 2002.

MUATA, K.; BRYSON, O.; KO, M. Exploring the relationship between information technology investments and firm performance using regression splines analysis. Information \& Management xxx xxx-xxx, 2003.

NOLAN, R. L. Managing the crises in data processing. Harvard Business Review, v. 57, n. 2, p. 115-126, Mar./Apr., 1979.
PEREZ, B. E. Information systems investment decisions in business practice: the Spanish case. European Journal of Information Systems, n. 7, p. 202-209, 1998.

PROUDLOCK, M. J., PHELPS, B.; GAMBLE, P.R. Is decision-making: a study in information-intensive firms. Journal of Information Technology, n. 13, p. 55-66, 1998.

RANGANATHAN, C.; KANNABIRAN, G Effetive management of information systems function: an exploratory study of Indian organizations. International Journa of Information Management, n. 24, p. 247266, 2004.

RONDEAU, P. J.; RAGU-NATHAN, T. S . Vonderembse, M. A. How involvement, is management effectiveness, and end- user computing impact is performance in manufacturing firms. Information $\mathcal{G}$ Management, xx:xx-xx, 2005.

SCHELP, D. Brasil High-Tech. Revista VEJA, n. 1811 , p. 88,16 jul. 2003.

STATSOFT, Inc. STATISTICA: data analysis software system, version 6, 2001. $1 \mathrm{CD}$.

SUlLIVAN, C. H. Systems Planning in the Information Age. Sloan Management Review, winter p. 3-12, 1985.

TEO, T. S. H.; ANG, J. S. K. An examination of major is planning problems. International Journal of Information Management, n. 21(6), p. 457-470, 2001
TEO, T. S. H.; ANG, J. S. K. Critical success factor in the alignment of IS plans with business plans. International Journal of Information Management, n. 19, p. 173 185, 1999.

TEO, T. S. H.; KING, W. R. Integration between Business Planning and Information Systems Planning: Na Evolutionary-Contingency Perspective. Journal of Management Information Systems, n. 14(1), p. 185-214, 1997.

THONG, J. Y. L. An Integrated model of Information Systems Adoption in Small Business. Journal of Management Information Systems, n. 15(4), p. 187-214, 1999.

TIERNAN, C.; PEPPARD, J. Information technology: of value or a vulture. $E u$ ropean Management Journal. n. 22(6), p. 609-623, 2004.

\section{Agradecimentos}

Os autores agradecem ao Conselho Nacional de Desenvolvimento Científico e Tecnológico - CNPq.

\section{- Sobre os autores}

\section{Ana Paula Cabral Seixas Costa}

UFPE - Universidade Federal de Pernambuco

Profa. Adjunto Departamento de Engenharia de Produção

End.: Av. Acadêmico Hélio Ramos s/n, Centro de Tecnologia e Geociências, Prédio Administrativo 9o Andar - CEP 50670-901

Tel.: (81) 2126-8728

E-mail: apcabral@ufpe.br

\section{José Gilson de Almeida Teixeira Filho}

UFPE - Universidade Federal de Pernambuco

Doutorando em Ciência da Computação/Mestre em Engenharia de Produção

End.: Rua Francisco de Barros Barreto, 198, ap. 201 - CEP 51021-550

Tel.: (81) 9192-0810

E-mail: jgtfilho@cin.ufpe.br

\section{Maisa Mendonça Silva}

UFPE - Universidade Federal de Pernambuco

Mestranda em Engenharia de Produção

End.: Rua Simão Mendes, 92, ap.401 - CEP 52050-110

Tel.: (81) 8815-6488

E-mail: maisa@ufpe.br 
Quadro 1: Pesquisa sobre o perfil das empresas em relação ao uso de sistemas de informação (si) e tecnologia da informação (TI).

\begin{tabular}{|c|c|c|}
\hline \multicolumn{3}{|l|}{ IDENTIFIGAÇÃO DO GESTOR: } \\
\hline Nome Completo: & Idade: & Sexo: ( ) Masculino ( ) Feminino \\
\hline \multicolumn{3}{|l|}{ Nível de Formação: } \\
\hline ( ) Ensino Fundamental - 10 Grau & ( ) Ensino Médio - 2o Grau & ( ) Ensino Profissional - Técnico \\
\hline ( ) Graduação & ( ) Especialização & ( ) Mestrado Acadêmico \\
\hline ( ) Mestrado Profissionalizante & \multicolumn{2}{|l|}{ ( ) Doutorado } \\
\hline \multicolumn{3}{|l|}{ Instituiç̧ão: } \\
\hline \multicolumn{3}{|l|}{ Nome do Curso: } \\
\hline ( ) Em andamento & \multicolumn{2}{|c|}{ ( ) Não concluído } \\
\hline \multicolumn{3}{|c|}{ Em qual das seguintes faixas se encaixa o seu salário atual? } \\
\hline ( ) Até $\mathrm{R} \$ 1.000,00$ & ( ) De $R \$ 1.000,01$ a $R \$ 2.000,00$ & ( ) De $R \$ 2.000,01$ a $R \$ 4.000,00$ \\
\hline ( ) De $R \$ 4.000,01$ a $R \$ 8.000,00$ & ( ) De $\mathrm{R} \$ 8.000,01$ a $\mathrm{R} \$ 16.000,00$ & ( ) Mais de $\mathrm{R} \$ 16.000,01$ \\
\hline \multicolumn{3}{|c|}{ Qual é a sua percepção como gestor em relação à Tecnologia da Informação (TI) na empresa? MÚLTIPLA ESCOLHA } \\
\hline $\begin{array}{l}\text { ( ) Vê a TI como melhoria interna para os } \\
\text { processos }\end{array}$ & ( ) Vê a TI como diferencial competitivo & ( ) Vê a Tl como facilitador da comunicação \\
\hline ( ) Vê a TI como despesa & \multicolumn{2}{|l|}{ ( ) Outros: } \\
\hline \multicolumn{3}{|l|}{ IDENTIFICAÇÃO DA EMPRESA: } \\
\hline \multicolumn{2}{|l|}{ Razão Social: } & Site: \\
\hline \multicolumn{2}{|l|}{ Nome Fantasia: } & E-mail: \\
\hline CNPJ: & CEP: & Ano de Fundação: \\
\hline Ramo de Atividade: & ( ) Indústria & ( ) Serviço \\
\hline \multicolumn{3}{|l|}{ Setor de Atividade (ex.: indústria alimentícia): } \\
\hline Tipo de Empresa: & ( ) Pública & ( ) Privada \\
\hline Tipo de Capital: & ( ) Internacional & Nacional e Internacional \\
\hline Em qual faixa melhor se enquadra o faturament & bruto anual da empresa? & \\
\hline ( ) Abaixo de $\mathrm{R} \$ 500.000,00$ & ( ) De $\mathrm{R} \$ 500.000,01$ a $\mathrm{R} \$ 1.000 .000,00$ & ( ) De $\mathrm{R} \$ 1.000 .000,01$ a $\mathrm{R} \$ 5.000 .000,00$ \\
\hline ( ) De $\mathrm{R} \$ 5.000 .000,01$ a $\mathrm{R} \$ 10.000 .000,00$ & ( ) De $\mathrm{R} \$ 10.000 .000,01$ a $\mathrm{R} \$ 50.000 .000,00$ & ( ) Acima de $\mathrm{R} \$ 50.000 .000,00$ \\
\hline Número de Funcionários: & & \\
\hline Número de Computadores: & & \\
\hline 1. Sua empresa tem cultura de usar sistemas & de informação? & \\
\hline ( ) Não & ( ) Em fase inicial de implantação & \\
\hline 2. Qual o nível de dependência da sua empresa & em relação ao uso de sistemas de informações & , na sua visão? \\
\hline ( ) Médio & ( ) Alto & \\
\hline 3. Quanto a empresa investe anualmente em S & I/TI: & \\
\hline ( ) Abaixo de $\mathrm{R} \$ 50.000,00$ & ( ) De $\mathrm{R} \$ 50.000,01$ a $\mathrm{R} \$ 100.000,00$ & ( ) Je $\mathrm{R} \$ 100.000,01$ a $\mathrm{R} \$ 500.000,00$ \\
\hline ( ) De $\mathrm{R} \$ 500.000,01$ a $\mathrm{R} \$ 1.000 .000,00$ & ( ) De $\mathrm{R} \$ 1.000 .000,01$ a $\mathrm{R} \$ 5.000 .000,00$ & ( ) Acima de $\mathrm{R} \$ 5.000 .000,00$ \\
\hline 4. Qual o nível de automação que a empresa sc & encontra, ou seja, o nível em que os processo & s estão informatizados? \\
\hline ( ) Médio & ( ) Alto & \\
\hline 5. A empresa utiliza alguma metodologia para & planejamento de sistemas de informação? Qual & \\
\hline ( ) Não & Qual: & \\
\hline 6. Qual o nível de satisfação dos clientes em $\mathbf{r}$ & lação aos serviços prestados pela sua empres & \\
\hline ( ) Razoável & ( ) Bom & \\
\hline 7. Como você avaliaria a disponibilidade das in & ormações para auxiliar uma tomada de decisão & da diretoria na sua empresa? \\
\hline ( ) Médio acesso & ( ) Fácil acesso & \\
\hline
\end{tabular}




\begin{tabular}{|c|c|c|}
\hline \multicolumn{3}{|c|}{ 8. Qual o critério utilizado pela sua empresa para investir em SI/TI? MÚLTIPLA ESCOLHA } \\
\hline ( ) Baseado no que os concorrentes investem & ( ) Critérios técnicos & ( ) Critérios financeiros \\
\hline ( ) Tendência de mercado & \multicolumn{2}{|l|}{ ( ) Outros: } \\
\hline \multicolumn{3}{|c|}{ 9. Sua empresa utiliza algum software como: MÚLTIPLA ESCOLHA } \\
\hline ( ) Data Mining & ( ) ERP & ( ) CRM \\
\hline ( ) Data Warehouse & ( ) Outros: & \\
\hline \multicolumn{2}{|c|}{ 10. O uso de SI/TI nos produtos e serviços da sua empresa procura melhorar para o cliente: } & MÚLTIPLA ESCOLHA \\
\hline ( ) Rapidez & ( ) Qualidade & ( ) Custo \\
\hline ( ) Confiabilidade/Credibilidade & ( ) Flexibilidade & ( ) Outros: \\
\hline \multicolumn{3}{|c|}{ 11. Como a empresa decide o investimento em Sistemas de Informação? MARQUE APENAS UMA } \\
\hline $\begin{array}{l}\text { ( ) Utiliza uma metodologia de planejamento de } \\
\text { investimento }\end{array}$ & $\begin{array}{l}\text { ( ) Decide baseada apenas em experiências } \\
\text { passadas }\end{array}$ & ( ) Utiliza ferramentas de apoio a decisão \\
\hline ( ) Nenhuma metodologia é utilizada & \multicolumn{2}{|l|}{ ( ) Outros: } \\
\hline \multicolumn{3}{|c|}{ 12. Em que estágio sua empresa encontra-se em relação a utilização de SI/TI? MARQUE APENAS UMA } \\
\hline $\begin{array}{l}\text { ( ) Poucas pessoas usam o computador e } \\
\text { somente para atender necessidades básicas } \\
\text { da organização }\end{array}$ & $\begin{array}{l}\text { ( ) Muitas pessoas usam o computador } \\
\text { sem gerenciamento e padronização de } \\
\text { ferramentas de tecnologia }\end{array}$ & $\begin{array}{l}\text { ( ) Existe controle no uso dos hardwares e } \\
\text { softwares com padronização e metodologias } \\
\text { claras }\end{array}$ \\
\hline $\begin{array}{l}\text { ( ) Integração dos sistemas existentes, } \\
\text { compartilhamento de dados e aplicações }\end{array}$ & $\begin{array}{l}\text { ( ) Gerenciamento não só dos hardwares } \\
\text { e softwares e também dos recursos de } \\
\text { informação }\end{array}$ & $\begin{array}{l}\text { ( ) Planejamento bem estabelecido e } \\
\text { alinhamento dos sistemas de informação } \\
\text { com a organização }\end{array}$ \\
\hline \multicolumn{3}{|c|}{ 13. Qual tipo de Sistema Operacional de rede é adotado na sua empresa? MÚLTIPLA ESCOLHA } \\
\hline ( ) Novell & ( ) Windows & ( ) Unix \\
\hline ( ) Linux & ( ) Outros: & \\
\hline \multicolumn{3}{|c|}{ 14. Que tipo de banco de dados sua empresa utiliza? MÚLTIPLA ESCOLHA } \\
\hline ( ) ORACLE & ( ) $\mathrm{DB} 2$ & ( ) Microsoft ACESS \\
\hline ( ) SQL SERVER & ( ) Outros: & \\
\hline \multicolumn{3}{|c|}{ 15. Que linguagens de programação sua empresa utiliza? MÚLTIPLA ESCOLHA } \\
\hline ( ) DELPHI & ( ) JAVA & ( ) PHP \\
\hline ( ) ASP & ( ) Outras: & \\
\hline \multicolumn{3}{|c|}{ 16. Em relação à internet, a sua empresa possui: MÚLTIPLA ESCOLHA } \\
\hline ( ) Portal Corporativo & ( ) Portal E-commerce & ( ) Site Institucional \\
\hline ( ) Intranet e/ou Extranet & \multicolumn{2}{|l|}{ ( ) Outros: } \\
\hline \multicolumn{3}{|c|}{ Deseja receber o resultado desta pesquisa por e-mail? } \\
\hline ( ) Não & \multicolumn{2}{|l|}{ Seu e-mail: } \\
\hline
\end{tabular}

